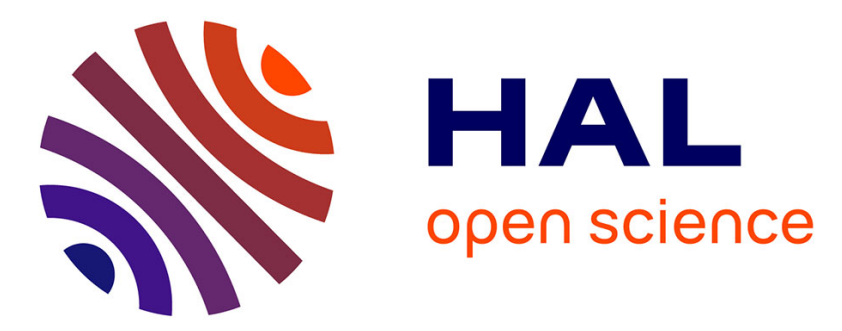

\title{
Modeling and experiments of high speed magnetic micromanipulation at the air/liquid interface
}

\author{
Mohamed Dkhil, Aude Bolopion, Stéphane Régnier, Michaël Gauthier
}

\section{To cite this version:}

Mohamed Dkhil, Aude Bolopion, Stéphane Régnier, Michaël Gauthier. Modeling and experiments of high speed magnetic micromanipulation at the air/liquid interface. RSJ International Conference on Intelligent Robots and Systems, Sep 2014, Chicago, IL, United States. 10.1109/IROS.2014.6943222 . hal-02927296

\section{HAL Id: hal-02927296 \\ https://hal.science/hal-02927296}

Submitted on 1 Sep 2020

HAL is a multi-disciplinary open access archive for the deposit and dissemination of scientific research documents, whether they are published or not. The documents may come from teaching and research institutions in France or abroad, or from public or private research centers.
L'archive ouverte pluridisciplinaire HAL, est destinée au dépôt et à la diffusion de documents scientifiques de niveau recherche, publiés ou non, émanant des établissements d'enseignement et de recherche français ou étrangers, des laboratoires publics ou privés. 


\title{
Modeling and experiments of high speed magnetic micromanipulation at the air/liquid interface
}

\author{
Mohamed Dkhil $^{(1,2)}$, Aude Bolopion ${ }^{1}$, Stéphane Régnier ${ }^{2}$, Member, IEEE, Michaël Gauthier ${ }^{1}$, Member, IEEE
}

\begin{abstract}
One of the greatest challenge in microrobotics is the development of miniaturized smart surfaces for a high speed conveying and positioning of micro-objects. This paper proposes a new approach where objects are situated at the air/liquid interface and are manipulated through magnetic fields. It demonstrates that a good repeatability and a high speed can be obtained. A physical modeling is presented to analyze the dynamic behavior of the micro-object. Experiments are performed to determine the physical parameters of the model and to attest the good repeatability of the motion for an object of size $100 \times 90 \times 25 \mu \mathrm{m}^{3}$. A good agreement between the physical model and the experimental measurement is demonstrated. Since the velocity of the micro-object can be 10 times higher at the air/liquid interface than in the liquid this approach represents a promising solution to design smart surfaces for a high throughput conveying of micro-objects.
\end{abstract}

\section{INTRODUCTION}

Smart surfaces are well studied solutions to manipulate, convey, and position objects of a few millimeters [1], [2], [3]. For industrial tasks such as the assembly and packaging of products automatic feeding of objects is crucial. To perform planar displacements a variety of actuation methods have been developed including electrostatic actuators [4], electromagnetic actuators [5] and air jets [6]. These methods enable to manipulate the objects with no direct contact which avoids damages of fragile components such as electronic cards [7].

The miniaturization of these smart surfaces in order to manipulate objects of a few micrometers would be an innovative solution with a wide range of application domains, from microassembly to cell manipulation. Several actuation techniques are available to perform 2D displacement of micrometer size objects, such as magnetophoresis [8], [9], [10]; dielectrophoresis [11], [12], [13]; laser beams [14], [15] or acoustic waves [16], [17]. However, one of the greatest challenge consists in providing an ultra-rapid surface for transportation. Currently, the proposed systems are either in ambiant environments or in liquids. At micrometer scale adhesion forces are a major issue in ambiant environments, and the repeatability of the manipulations is not guaranteed [18]. In a liquid medium the velocity is limited due to the viscous forces [19].

\footnotetext{
${ }^{1}$ Authors are with FEMTO-ST Institute CNRS UFC/ENSMM/UTBM Automatic Control and Micromechatronic Systems Department, 24 rue Alain Savary, 25000 Besançon, France. mohamed.dkhil@ femto-st.fr

${ }^{2}$ Authors are with Institut des Systèmes Intelligents et de Robotique (ISIR), Pierre et Marie Curie University, CNRS UMR 7222, 4 Place Jussieu, 75005 Paris, France.
}

Complementary to smart surfaces proposed in the literature, this paper proposes a planar magnetic micromanipulation principle. The micro-object is placed at the interface between two fluids, and the actuation is ensured by magnetic forces. The object is in equilibrium on the interface under the action of the surface tension and its weight and it moves on the surface with minimum friction. This approach enables to avoid adhesion issues and provides a high velocity motion. A model of the system is proposed, and the physical parameters, namely the drag coefficient and the magnetization, are identified. The repeatability and velocity are discussed and the dynamic model derived in this article can be used for closed loop control in future works.

This paper is organized as follows. In Section II, the dynamic behavior of the micro-object is modeled. The repeatability of the motion is evaluated in Section III based on experimental measurements. In Section IV, the physical parameters of the model are identified and its validity is discussed. The advantages and limitations of this approach are discussed in Section V. Conclusions and perspectives of this work are presented in Section VI.

\section{DYNAMIC MODEL}

Most of the magnetic actuation systems present similar designs. The modeling proposed in this paper is illustrated on a magnetic device composed of four electromagnets. The magnetic force produced induces the motion of the microobject on the air/liquid interface (see Figure 1). In this section a model of the motion of the object on a meniscus is derived. Some hypothesis will then be assumed to simplify the dynamic model and highlight the influence of the main physical parameters (drag coefficient, magnetization) on the micro-object behavior.

\section{A. Model of the motion of a micro-object on a air/liquid interface}

So that the object can be moved by magnetic fields it must be composed of a magnetic material. The microobject is then placed on the air/liquid interface. At this scale the workspaces are small (at most some millimeters). A meniscus appears on the reservoir, which must be taken into account while modeling the motion (see Figure 1). The shape of the meniscus can be concave surface depends on the material properties of the reservoir. It is defined by the function $f$ :

$$
z=f(x)
$$


It will be considered in the following that the motion of the object is on the $(x, z)$ plane.

The forces applied to the micro-object are: (i) the magnetic force $\vec{F}_{\text {mag }}$ generated by electromagnets, (ii) the weight $\vec{P}$ (iii), the resultant force of surface tension $\vec{T}$ and (iv) the drag force $\vec{F}_{v}$.

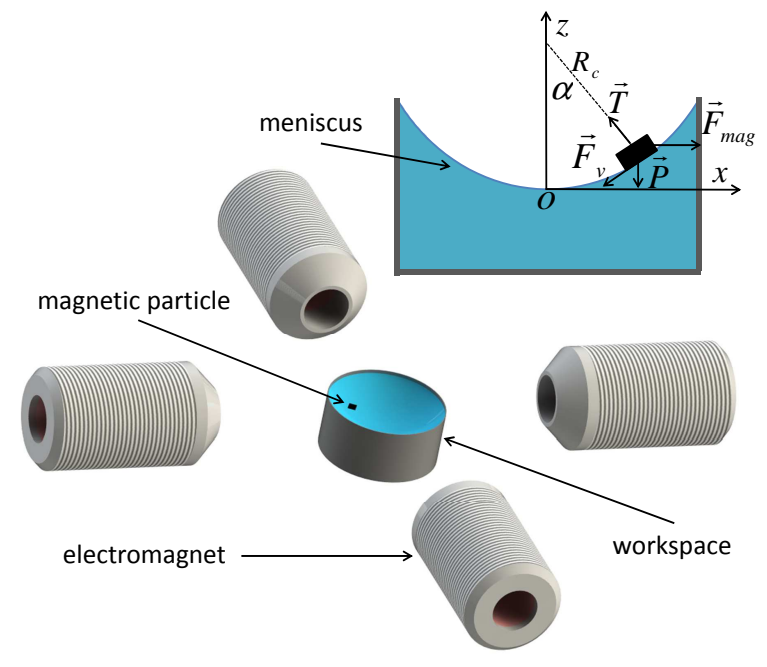

Fig. 1. Example of a planar magnetic device. The electromagnets control the motion of the micro-object on the air/liquid interface. A meniscus of radius of curvature $R_{c}$ is formed at the interface. $\alpha$ is the angle between the micro-object and the vertical direction. The magnetic force $\vec{F}_{m a g}$, the weight $\vec{P}$, the resultant force of surface tension $\vec{T}$ of the air/liquid interface and the drag force $\vec{F}_{v}$ are applied to the micro-object.

The magnetic force $\vec{F}_{\text {mag }}$ applied to a magnetic microobject is given by:

$$
\vec{F}_{\text {mag }}=V \cdot \nabla(\vec{M} \cdot \vec{B})
$$

where $V$ is the volume of the object, $\vec{M}$ is its magnetization and $\nabla \vec{B}$ is the magnetic field gradient produced by the magnetic source. The fluid flow is considered as a Stokes flow. The drag force is given by:

$$
\vec{F}_{v}=-h \vec{v}
$$

where $h$ is the drag coefficient and $\vec{v}$ is the velocity of the object.

The weight of the micro-object $\vec{P}$ is compensated by the resultant force of the surface tension $\vec{T}$ (see Figure 2). The equilibrium condition is given by:

$$
\gamma C \sin (\phi)=m g
$$

where $\gamma$ is the surface tension, $C$ is the perimeter of the object, $\phi$ is the contact angle between the micro-object and the liquid, $m$ is the mass of the object and $g=9.8 \mathrm{~m} \cdot \mathrm{s}^{-2}$ is the acceleration of the gravity. To evaluate the deformation of the meniscus due to the presence of the object the $\phi$ angle is evaluated numerically. A rectangular nickel object is considered (density: $\rho=8902 \mathrm{~kg} / \mathrm{m}^{3}$, size: 100x90x $25 \mu^{3}$ ) and the liquid is water (surface tension: $\gamma=7210^{-3} \mathrm{~N} / \mathrm{m}$ ). Based on Equation (4) the $\phi$ angle is about $7.1710^{-4} \mathrm{rad}$ which is negligible. The shape of the meniscus is not affected by the weight of the micro-object and Eq. (1) can be used despite the presence of the object.

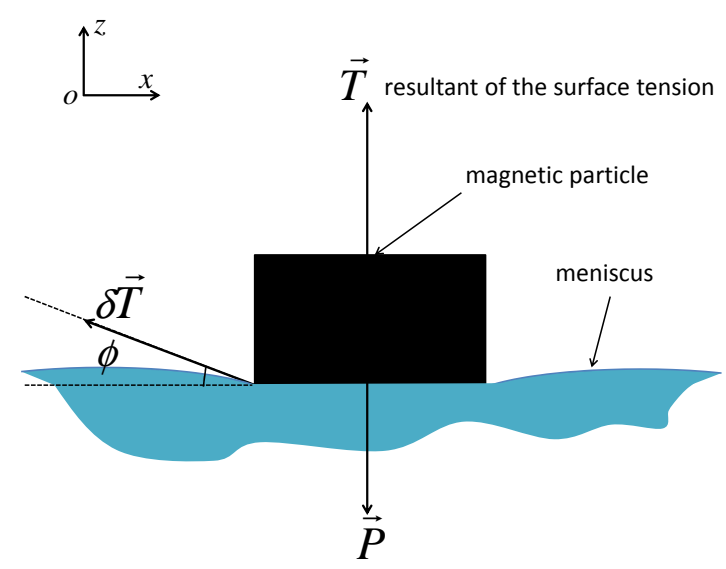

Fig. 2. Equilibrium of the magnetic micro-object on the meniscus. The resultant force of surface tension $\vec{T}$ compensates the weight $\vec{P}$. It can be shown that the contact angle $\phi$ between the micro-object and the liquid is negligible, therefore, the shape of the meniscus in the reservoir is not deformed by the weight of the micro-object.

Using the second Newton's law, the motion of the microobject can be expressed as follows:

$$
m \vec{a}=\vec{P}+\vec{F}_{m a g}+\vec{F}_{v}+\vec{T}
$$

where $\vec{a}$ is the acceleration of the micro-object. This equation is projected along $(o, \vec{x})$ and $(o, \vec{z})$ :

$$
\left\{\begin{array}{c}
m \ddot{x}=F_{\text {magx }}-h v \cos (\alpha)-T \sin (\alpha), \\
m \ddot{z}=F_{\text {magz }}-P-h v \sin (\alpha)+T \cos (\alpha), \\
z=f(x), \\
\tan (\alpha)=\frac{d f(x)}{d x},
\end{array}\right.
$$

where $\alpha$ is the angle between the vertical direction and the direction going from the center of the sphere tangent to the meniscus and the object (see Figure 1). The general behavior of the micro-object is given by Eq. (6). Some assumptions will now be made to highlight the influence of the physical parameters on its behavior.

\section{B. Simplified dynamic model}

In order to study the characteristics of this system a linear model is derived based on the following assumptions:

- The curvature of the meniscus is considered spherical, with a radius $R_{c}$. The position $x$ and $z$ are related to $R_{c}$ 
and the angle $\alpha$ by:

$$
\left\{\begin{array}{c}
x=R_{c} \sin (\alpha), \\
z=R_{c}(1-\cos (\alpha)),
\end{array}\right.
$$

- Only local displacements around the center of the workspace are considered. Thus $\alpha<<1$ and the approximations $\sin (\alpha) \approx \alpha$ and $\cos (\alpha) \approx 1$ are satisfied.

- Due to the small size of the object the inertial terms $m \ddot{x}$ and $m \ddot{z}$ can be neglected in Eq. (6).

The validity of these assumptions will be discussed in Section IV.

Based on the above assumptions the dynamic model simplifies to:

$$
h \dot{x}+\left(\frac{m g}{R_{c}}\right) x=F_{m a g}(x, I),
$$

where $I$ is the current in the electromagnets. To illustrate the motion of the object only one electromagnet will be considered in the following. It is considered that the magnetic force applied on the micro-object is directed along the $(o, \vec{x})$ axis. In order to define the expression of the magnetic force the magnetic field gradient must be determined. Experimental measurements are performed (see Figure 3). If the microobject is far from the electromagnet (further than $9 \mathrm{~mm}$ in this case) it experiences an almost constant magnetic field and magnetic field gradient. The magnetic field gradient $\nabla \vec{B}(I)$ and the magnetization $\vec{M}(I)$ depend only on the current $I$ set to the electromagnet. In the following this approximation will be made since the object will remain further than $9 \mathrm{~mm}$ away from the electromagnet.

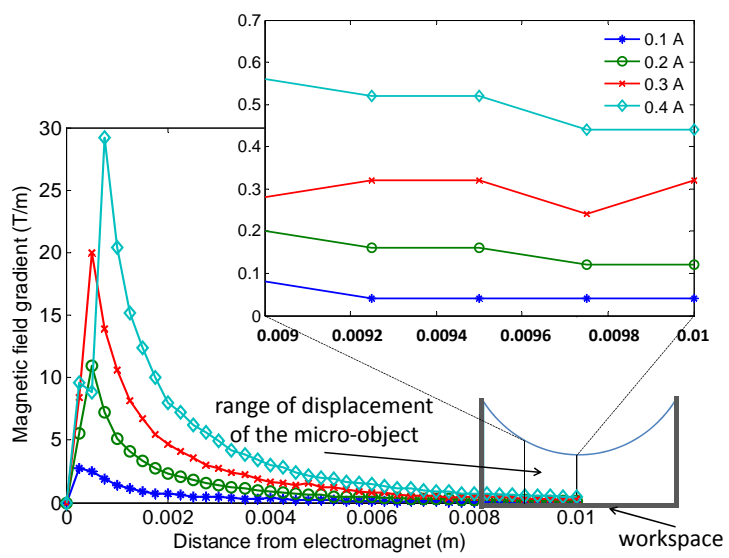

Fig. 3. Magnetic field gradient produced by one electromagnet along the $(o, x)$ axis. Measurements are made using a teslameter (F.W Bell, Model 7010) based on the use of an Hall probe. The magnetic field gradient is considered constant further than $9 \mathrm{~mm}$ away from the electromagnet. Thus, it is constant along the trajectory of the micro-object.
Based on the above results the simplified dynamic model is:

$$
h \dot{x}+\left(\frac{m g}{R_{c}}\right) x=V(M(I) \nabla B(I)) .
$$

The dynamic behavior of the micro-object is given by a first order differential equation whose solution depends on the shape of the meniscus.

The magnetic force attracts the object toward the electromagnet. While it moves on the meniscus the projection along the $(o, \vec{x})$ axis of the resultant force of surface tension and the magnetic force cancel out and the object reaches an equilibrium position noted $X_{\infty}$ (see Figure 4 ). When the magnetic force is switched off the object goes back to the center of the workspace under the action of gravity.

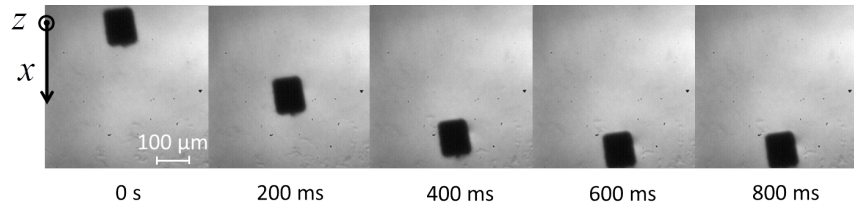

Fig. 4. Trajectory of the micro-object on the air/water interface. Only the first phase, when the magnetic force is activated, is shown here. The electromagnet is situated at the bottom of the images.

The permanent position $X_{\infty}$ obtained when the gravity and the magnetic force cancel out is expressed as follows:

$$
X_{\infty}=\frac{R_{c}(M(I) \nabla B(I))}{\rho g},
$$

where $m=\rho V$ with $\rho$ the density of the object. The response time $\tau$ to reach the permanent position is:

$$
\tau=\frac{h R_{c}}{m g}
$$

The determination of these two parameters $\left(X_{\infty}\right.$ and $\left.\tau\right)$ allow the identification of physical parameters, namely the drag coefficient $h$ and the magnetization of the material $M$. They will be identified based on experimental measurements. To ensure the validity of this identification the repeatability of the system is first evaluated in the next section.

\section{EVALUATION OF THE REPEATABILITY FOR HIGH SPEED MICROMANIPULATION}

Several factors can affect the repeatability of the system among which external disturbances such as dust and vibrations, or the shape of the meniscus. They are discussed in the following for objects of different sizes.

\section{A. Experimental setup}

The magnetic device is composed of four in-plane electromagnets (see Figure 5). Only one electromagnet will be activated to move the micro-object. To minimize the mechanical noise the magnetic device is placed on an antivibration base (Newport SG Breadboard). Control of the current in the electromagnet is performed by a custom software that communicates with an electronic amplifier through a 
National Instrument card (PCI 6733). A rectangular microobject composed of electrodeposited nickel (see [20] for the fabrication process) is placed on an air/water interface (distilled water is used). An image-processing algorithm able to detect the micro-object position in real time has been developed based on thresholding techniques. Image acquisition is done using a Photon focus camera with a frame rate of about $70 \mathrm{fps}$.

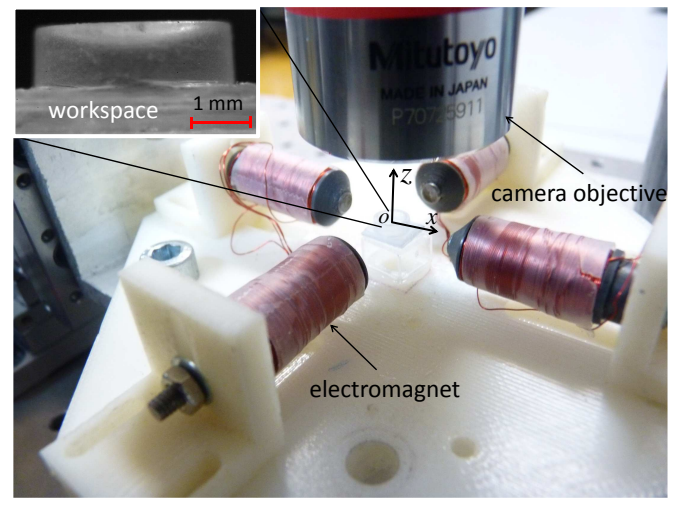

Fig. 5. Experimental setup. The inlet represents the cylindrical workspace used (diameter: $4 \mathrm{~mm}$ ). The meniscus formed is spherical. Its radius is measured on the image $\left(R_{c}=10.5 \mathrm{~mm}\right)$.

\section{B. Repeatability with a constant meniscus}

To determine the impact of the environmental conditions the same experiment is performed five times. The electromagnet is powered with the same actuation signal (step of current of magnitude $I=0.47$ A during $2 \mathrm{~s}$ ). The size of the micro-object used is $100 \times 90 \times 25 \mu \mathrm{m}^{3}$, and the radius of curvature of the meniscus $R_{c}$ is estimated to $10.5 \mathrm{~mm}$ from a side view of the setup. Figure 6 presents the position response of the system for each of the five trials. The microobject moves under the action of the magnetic force to reach a permanent position at around $X_{\infty}=300 \mu \mathrm{m}$ and comes back to the center of the meniscus under the action of the gravity. The standard deviation of the response time $\sigma_{(\tau)}$ is a few milliseconds while the one of the permanent position $\sigma_{\left(X_{\infty}\right)}$ is a few micrometers. The behavior of the micro-object is thus repeatable, which offers the possibility to use the air/water interface for planar magnetic micromanipulation.

\section{Impact of the meniscus variations on the repeatability}

The curvature of the meniscus is highly sensitive to the filling operation. It is thus not possible to guarantee a constant radius of curvature since the reservoir has to be re-filled periodically due to the evaporation of the water. To limit the inaccuracies the following procedure has been defined: the workspace is completely filled with distilled water. The micro-object is placed on the center of the workspace. A heating element is placed 15 minutes under the workspace to evaporate partially the water and generate

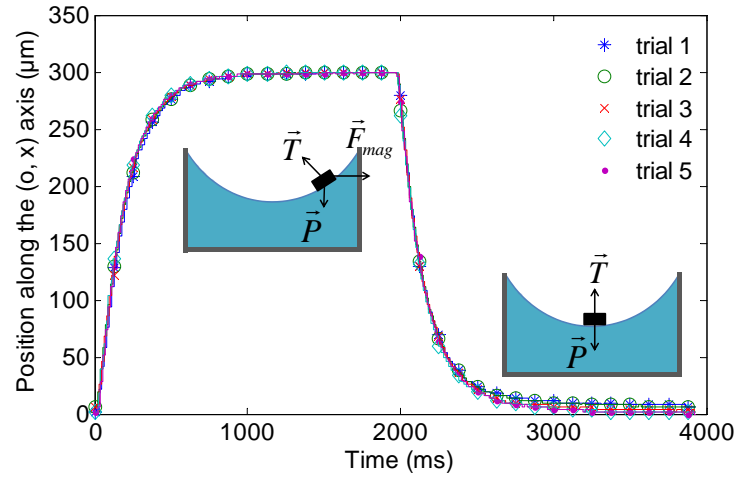

Fig. 6. Position response measured for five different trials in the same conditions. The object size is $100 \times 90 \times 25 \mu^{3}$, the radius of curvature of the meniscus is $10.5 \mathrm{~mm}$ and a current $I=0.47 \mathrm{~A}$ is applied for $2 \mathrm{~s}$. The average values of the permanent position and the response time are respectively $\overline{X_{\infty}}=300 \mu \mathrm{m}$ and $\bar{\tau}=187 \mathrm{~ms}$ and the standard deviations are $\sigma_{\left(X_{\infty}\right)}=1 \mu \mathrm{m}$ and $\sigma_{(\tau)}=6 \mathrm{~ms}$.

the final meniscus shape. To evaluate the impact of the variability of the meniscus geometry on the repeatability of the micro-object behavior, five experiments are performed. After each of them the workspace is emptied and re-filled. The same experimental conditions than in Sec. III-B are used. Results are given in Figure 7. The average value and standard deviations of $\tau$ and $X_{\infty}$ are respectively $\bar{\tau}=174 \mathrm{~ms}$, $\sigma_{(\tau)}=3 \mathrm{~ms}$ and $\overline{X_{\infty}}=283 \mu \mathrm{m}, \sigma_{\left(X_{\infty}\right)}=12 \mu \mathrm{m}$. The standard deviation of the permanent position represents $4 \%$ of the average value. It is thus not possible to ensure a precise positioning of the micro-object due to the sensibility of the permanent position to the curvature of the meniscus. A closed loop control would be necessary if precise positioning is needed. The dynamic model derived in this paper (Sec. IV) can be used to perform this control.

\section{Impact of the micro-object size on the repeatability}

To evaluate the impact of the size of the object on its behavior experiments are performed with a smaller object $\left(60 \times 50 \times 25 \mu^{3}\right)$. The same experimental conditions than in Sec. III-B are used. Table I compares the results for both the large and the small objects. The permanent position $X_{\infty}$ is similar in both cases. Indeed from Eq. (10) it can be seen that this parameter does not depend on the size of the micro-object. Its standard deviation does not vary significantly between large and small objects. On the contrary the response time $\bar{\tau}$ is larger for smaller objects as expressed in Eq. (11). It is also less repeatable for small micro-objects. One explanation can be that their motion is more sensitive to the mechanical vibrations because of the reduction of the inertia. Additional experiments have been performed on a $30 \times 20 \times 25 \mu^{3}$ size object but its behavior is not repeatable.

The reduction of the object size decreases the repeatability of the dynamic behavior. Thus the approach of the micromanipulation using air/liquid interface and magnetic actuation 


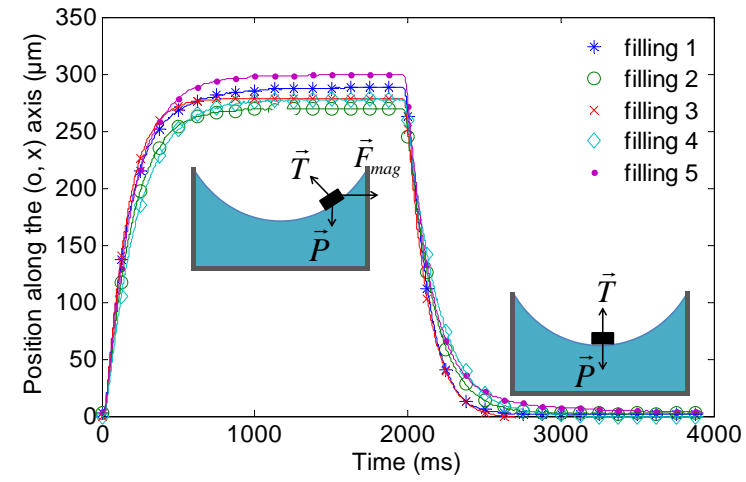

Fig. 7. Position response for five different trials. The workspace is re-filled between each trial, thus the shape of the meniscus is slightly different for each trial. The object size is $100 \times 90 \times 25 \mu \mathrm{m}^{3}$, a current $I=0.47 \mathrm{~A}$ is applied for $2 \mathrm{~s}$. The average values of the permanent position and the response time are respectively $\overline{X_{\infty}}=283 \mu \mathrm{m}$ and $\bar{\tau}=174 \mathrm{~ms}$ and the standard deviations are $\sigma_{\left(X_{\infty}\right)}=12 \mu \mathrm{m}$ and $\sigma_{(\tau)}=3 \mathrm{~ms}$.

TABLE I

IMPACT OF THE SIZE OF THE OBJECT ON THE REPEATABILITY. $I=0.47 \mathrm{~A}, R_{c}=10.5 \mathrm{~mm}$, Constant SIGNIFIES "REPEATABILITY WITH CONSTANT MENISCUS", Variable SIGNIFIES "IMPACT OF THE MENISCUS VARIATIONS ON THE REPEATABILITY".

\begin{tabular}{|c|c|c|c||c|c|}
\hline Size & Meniscus & $\bar{\tau}$ & $\sigma_{(\tau)}$ & $\bar{X}_{\infty}$ & $\sigma_{\left(X_{\infty}\right)}$ \\
\hline$\left(\mu \mathrm{m}^{3}\right)$ & - & $(\mathrm{ms})$ & $(\mathrm{ms})$ & $(\mu \mathrm{m})$ & $(\mu \mathrm{m})$ \\
\hline \multirow{2}{*}{$100 \times 90 \times 25$} & Constant & 187 & 6 & 300 & 1 \\
\cline { 2 - 6 } & Variable & 174 & 3 & 283 & 12 \\
\hline \multirow{2}{*}{$60 \times 50 \times 25$} & Constant & 433 & 12 & 296 & 1 \\
\cline { 2 - 6 } & Variable & 450 & 31 & 287 & 16 \\
\hline
\end{tabular}

will be limited to objects larger than $50 \mu \mathrm{m}$.

\section{IDENTIFICATION AND VALIDATION OF THE MODEL}

To control the position of the micro-object $x$, the adequate current $I$ must be sent to the electromagnet to produce a given force $F_{m a g}$ (see Figure 8). The analytical relations between $I$ and $F_{m a g}$ and between $F_{m a g}$ and $x$ are known (Eq. (2), (8)). However the value of several key parameters, namely the magnetization of the micro-object and the drag coefficient, must be determined to get a numerical model that could be used for closed loop control. This section presents a method for the identification of these parameters based on experimental measurements. Data from the previous section are used and additional experiments are performed to obtain measurements for several values of $I$.

\section{A. Identification of the magnetic behavior}

The magnetization $M$ of the micro-object is a key parameter, which varies a lot depending on the properties of the object, such as its fabrication process. It is thus not easy to obtain its value from the literature. However it can be identified based on Eq. (10) and on the experimental measurements. Table II presents its value for several magnitude

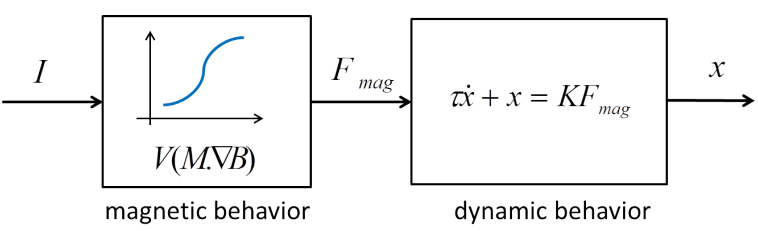

Fig. 8. Relation between the input of the system (current $I$ set to the electromagnet) and its output (position $x$ of the micro-object).

TABLE II

VALUES OF THE PERMANENT POSITION $X_{\infty}$, THE MAGNETIC FIELD GRADIENT $\nabla B_{\text {moy }}$ IN THE WORKSPACE AND THE MAGNETIZATION $M$ FOR DIFFERENT VALUES OF THE CURRENT. $R_{c}=10.5 \mathrm{~mm}$, $\rho_{\text {Nickel }}=8902 \mathrm{~kg} / \mathrm{m}^{3}$.

\begin{tabular}{|c|c|c|c|}
\hline$I(\mathrm{~A})$ & $X_{\infty}(\mu \mathrm{m})$ & $\nabla B_{\text {moy }}(\mathrm{T} / \mathrm{m})$ & $M(\mathrm{~A} / \mathrm{m})$ \\
\hline 0.26 & 52 & 0.26 & 1661 \\
\hline 0.32 & 98 & 0.33 & 2467 \\
\hline 0.40 & 157 & 0.39 & 3344 \\
\hline 0.47 & 283 & 0.48 & 4898 \\
\hline
\end{tabular}

of current. The identified values depend on $I$ but remain below the saturation of the nickel material $\left(4.710^{5} \mathrm{~A} / \mathrm{m}\right.$ according to [21]). The magnetic force is non linear with respect to $I$ and can be expressed by the following expression for the typical range of current used for this magnetic device $0.26 \mathrm{~A}<I<0.47 \mathrm{~A}$ :

$$
F_{\text {mag }}(I)=V\left(16200 I^{2}-2900 I+95\right)
$$

This relation enables to derive the analytical relation between the input current $I$ and the magnetic force applied to the micro-object (first bloc of Figure 8). In the case this model is used for closed loop control this relation can be easily inverted.

\section{B. Identification of the dynamic behavior - drag coefficient}

The dynamic behavior of the micro-object is ruled by the response time expressed by Eq. (11) and the static gain expressed by the ratio $K=\frac{R_{c}}{m g}$. These relations involve several physical parameters, namely $R_{c}, m$ and $h$. The radius of curvature $R_{c}$ can be estimated with side view images of the setup. In addition the mass of the object $m$ can be calculated through the value of the density of the material founded in literature and the dimensions of the object measured under a microscope. The drag coefficient is however an unknown parameter in the dynamic model, which is not easily found in the literature since it varies greatly depending on the shape of the object. The value of this parameter is calculated in Table III for several values of the input current. A predicted by Eq. (11) its value does not depend on the input current. The average value of the drag coefficient is $h=3.3910^{-7} \mathrm{~kg} / \mathrm{s}$. It can be noted that this coefficient does not depend on the velocity of the micro-object. The computation of the Reynolds number $R_{e}$ indeed confirms the assumption of Stokes flow since $R_{e}<1$. The identified value 
TABLE III

EXPERIMENTAL MEASUREMENTS OF THE RESPONSE TIME $\tau$ FOR DIFFERENT INPUT CURRENT $I$. THE DRAG COEFFICIENT $h$ AND THE REYNOLDS NUMBER $R_{e}$ ARE COMPUTED BASED ON THESE MEASURES FOR $R_{c}=10.5 \mathrm{~mm}, \rho_{\text {Nickel }}=8902 \mathrm{~kg} / \mathrm{m}^{3}$.

\begin{tabular}{|c|c|c|c|}
\hline$I(\mathrm{~A})$ & $\tau(\mathrm{ms})$ & $h(\mathrm{~kg} / \mathrm{s})$ & $R_{e}$ \\
\hline 0.26 & 194 & $3.63 \quad 10^{-7}$ & 0.15 \\
\hline 0.32 & 178 & $3.32 \quad 10^{-7}$ & 0.17 \\
\hline 0.40 & 181 & $3.37 \quad 10^{-7}$ & 0.16 \\
\hline 0.47 & 174 & $3.24 \quad 10^{-7}$ & 0.17 \\
\hline
\end{tabular}

of the drag coefficient gives access to the numerical model of the dynamic behavior of the micro-object.

\section{Validation of the model}

Using the parametric approximation of the magnetic force determined in Sec. IV-A and the drag coefficient $h=$ $3.3910^{-7} \mathrm{~kg} / \mathrm{s}$ identified in Sec. IV-B the dynamic model (Eq. (9)) of the micro-object behavior is simulated. Figure 9 provides a comparison between the simulation and the experimental measurements of the position response for several current values. The trends of the simulated and experimental plots are similar. The differences that can be noted might come from the approximation of the magnetic force in Eq. (12).

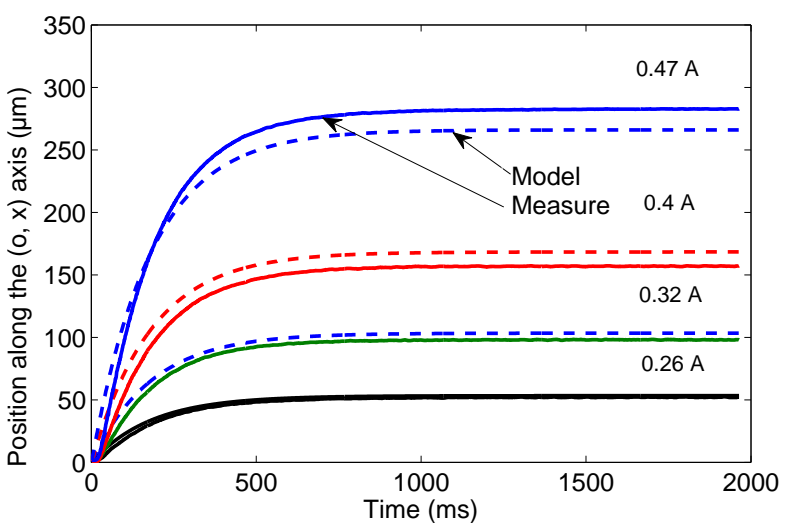

Fig. 9. Comparison between the simulated and the experimental responses. The numerical values used in the simulation are the following: $R_{c}=$ $10,5 \mathrm{~mm}, \rho=8902 \mathrm{~kg} / \mathrm{m}^{3}$.

The assumptions assumed in Sec. II-B are indeed verified:

- From side view images of the reservoir it has been verified that the shape of the meniscus is spherical (see Figure 5).

- The magnetic force induces only local displacements around the center of the workspace. From the experimental measurements it has been estimated that the displacement to reach the permanent position $X_{\infty}$ is around 35 times smaller than the value of the radius of curvature $R_{c}$.

- The plots of the position measurements (see Figure 6 for example) are typical of a first order system, for which the velocity is not continuous. They confirm that the inertia of the object can be neglected.

\section{Discussion}

This approach of micromanipulation is limited to planar manipulation. In addition, the repeatability of the dynamic behavior is not ensured for objects smaller than $50 \mu \mathrm{m}$, most probably due to mechanical vibrations and possible dust particles that can be present on the liquid. In future works the source of this limitation should be identified and this issue should be addressed to generalize this approach to smaller micro-objects.

On the other hand, compared to current approaches in ambiant environments, the proposed magnetic micromanipulation principle avoids the issues related to adhesion forces. To compare the velocity that can be reached on the air/liquid interface to the one in liquid a numerical simulation has been performed using Comsol Multiphysics. The viscous coefficient of the micro-object of size $100 \times 90 \times 25 \mu^{3}$ immersed inside water is equal to $2.210^{-6} \mathrm{~kg} / \mathrm{s}$. This value is about 10 times larger than the identified value $h$ which means (see Eq. (9)) that the velocity of the micro-object on the air/water interface should be 10 times larger than inside water. The approach proposed in this paper is a promising solution for high speed conveying of micro-objects.

\section{CONCLUSION}

This paper demonstrates the ability to move a microobject on a air/liquid interface using magnetic actuation. A model of the dynamic behavior of the micro-objects on the meniscus has been derived, and the physical parameters that rule the behavior of the objects (their magnetization and their drag coefficient) have been identified. Experimental measurements demonstrate the repeatability of the motion for objects larger than $50 \mu \mathrm{m}$ (the variation of the permanent position of an object of $100 \times 90 \times 25 \mu \mathrm{m}^{3}$ for a constant meniscus represents about $1 \%$ of its size). This good repeatability is mainly due to the absence of adhesion forces. In addition the velocity of the micro-object at the air/water interface can be 10 times higher than in water. This work represents a promising solution for the design of magnetic device for high throughput conveying of micro-objects. However, if a precise positioning is required, a closed loop control must be implemented due to the sensitivity of the system to the radius of curvature of the meniscus.

This work is only the first step towards high speed/precise planar magnetic micromanipulation at microscale. In future works closed loop control will be implemented based on the model derived in this paper. The velocity of the micro-object will be increased by decreasing the distance between the electromagnets and the workspace, which will also require a more accurate modeling of the magnetic force that could not be considered as constant anymore. 


\section{Acknowledgements}

This work has been supported by the French Agence Nationale de la Recherche, through the LEMA project (contract "ANR 12 BS03 007 01"), by the Labex ACTION project (contract "ANR-11-LABX-01-01"), by the "Région Franchecomté" and by the French RENATECH network and its FEMTO-ST technological facility.

\section{REFERENCES}

[1] I. Georgilas, A. Adamatzky, and C. Melhuish, "Towards an intelligent distributed conveyor," in Advances in Autonomous Robotics. Springer, 2012, pp. 457-458.

[2] A. Delettre, G. J. Laurent, Y. Haddab, and N. Le Fort-Piat, "Robust control of a planar manipulator for flexible and contactless handling," Mechatronics, vol. 22, no. 6, pp. 852-861, 2012.

[3] D. El Baz, V. Boyer, J. Bourgeois, E. Dedu, and K. Boutoustous, "Distributed part differentiation in a smart surface," Mechatronics, vol. 22, no. 5, pp. 522-530, 2012.

[4] D. V. Dao, P. H. Pham, and S. Sugiyama, "A novel micro transportation system with fast movement of a micro container based on electrostatic actuation and a ratchet mechanism," Journal of Micromechanics and Microengineering, vol. 20, no. 11, p. 115026, 2010.

[5] M. U. Khan, N. Bencheikh, C. Prelle, F. Lamarque, T. Beutel, S. Büttgenbach et al., "A long stroke electromagnetic xy positioning stage for micro applications," IEEE/ASME Transactions on Mechatronics, pp. 1-10, 2011.

[6] A. Delettre, G. J. Laurent, L. Fort-Piat et al., "2-dof contactless distributed manipulation using superposition of induced air flows," in International Conference on Intelligent Robots and Systems, 2011, pp. 5121-5126.

[7] K. Park, S. Lee, J. Yi, S. Kim, Y. Kwak, and I. Wang, "Contactless magnetically levitated silicon wafer transport system," Mechatronics, vol. 6, no. 5, pp. 591-610, 1996.

[8] C. Pawashe, S. Floyd, E. Diller, and M. Sitti, "Two-dimensional autonomous microparticle manipulation strategies for magnetic microrobots in fluidic environments," IEEE Transactions on Robotics, pp. 467-477, 2012.

[9] M. Hagiwara, T. Kawahara, T. Iijima, Y. Yamanishi, and F. Arai, "High speed microrobot actuation in a microfluidic chip by levitated structure with riblet surface," in IEEE International Conference on Robotics and Automation, 2012, pp. 2517-2522.

[10] A. Chen, T. Byvank, W.-J. Chang, A. Bharde, G. Vieira, B. L. Miller, J. J. Chalmers, R. Bashir, and R. Sooryakumar, "On-chip magnetic separation and encapsulation of cells in droplets," Lab on a Chip, 2013.

[11] T. Yue, M. Nakajima, H. Tajima, and T. Fukuda, "Fabrication of microstructures embedding controllable particles inside dielectrophoretic microfluidic devices," International journal of advanced robotic systems, vol. 10, 2013.

[12] S.-W. Nam, S. H. Kim, J.-K. Park, and S. Park, "Dielectrophoresis in a slanted microchannel for separation of microparticles and bacteria," Journal of nanoscience and nanotechnology, vol. 13, no. 12, pp. 79937997, 2013.

[13] M. Kharboutly, M. Gauthier et al., "High speed closed loop control of a dielectrophoresis-based system." in IEEE International Conference on Robotics and Automation, 2013, pp. 1438-1443.

[14] X. Wang, S. Chen, C.-w. Kong, R. A. Li, and D. Sun, "Automated laser-induced cell fusion based on microwell array," in International Conference on Robotics and Automation, 2013, pp. 2795-2800.

[15] S. Chowdhury, A. Thakur, P. Svec, C. Wang, W. Losert, and S. K. Gupta, "Automated manipulation of biological cells using gripper formations controlled by optical tweezers," 2013.

[16] J. Lei, P. Glynne-Jones, and M. Hill, "Acoustic streaming in the transducer plane in ultrasonic particle manipulation devices," Lab Chip, vol. 13, no. 11, pp. 2133-2143, 2013.

[17] D. Foresti, M. Nabavi, M. Klingauf, A. Ferrari, and D. Poulikakos, "Acoustophoretic contactless transport and handling of matter," Bulletin of the American Physical Society, vol. 58, 2013.
[18] A. Ivan, G. Hwang, J. Agnus, N. Chaillet, and S. Régnier, "Nist micrororobotics challenge. magpier: The fastest mobile microrobots in the world," IEEE Robotics and Automation Magazine, vol. 20, pp. 63-70, 2013.

[19] M. Gauthier, S. Régnier, P. Rougeot, and N. Chaillet, "Analysis of forces for micromanipulations in dry and liquid media," Journal of Micromechatronics, vol. 3, no. 3-4, pp. 3-4, 2006.

[20] L. Wang, M. Dkhil, A. Bolopion, P. Rougeot, S. Régnier, and M. Gauthier, "Simulation and experiments on magnetic microforces for magnetic microrobots applications," The Third International Conference on Manipulation, Manufacturing and Measurement on the Nanoscale, 2013.

[21] K. Mulyokov, G. Korznikov, R. Abdulov, and R. Valiev, "Magnetic hysteretic properties of submicron grained nickel and their variations upon annealing," Journal of magnetism and magnetic materials, vol. 89 , no. 1-2, pp. 207-213, 1990. 